
internationales

vol. 20 - n² 2004

L'asile politique en Europe depuis l'entre-deux-guerres

\title{
Les camps d'étrangers depuis 1938 : continuité et adaptations
}

Du « modèle » français à la construction de l'espace Schengen

Olivier Clochard, Yvan Gastaut et Ralph Schor

\section{(2) OpenEdition}

\section{Journals}

Édition électronique

URL : https://journals.openedition.org/remi/968

DOI : 10.4000/remi.968

ISSN : $1777-5418$

Éditeur

Université de Poitiers

Édition imprimée

Date de publication : 1 septembre 2004

Pagination : $57-87$

ISBN : 2-911627-37-7

ISSN : 0765-0752

\section{Référence électronique}

Olivier Clochard, Yvan Gastaut et Ralph Schor, «Les camps d'étrangers depuis 1938 : continuité et adaptations ", Revue européenne des migrations internationales [En ligne], vol. 20 - n² | 2004, mis en ligne le 25 septembre 2008, consulté le 14 avril 2022. URL : http://journals.openedition.org/remi/968 DOI : https://doi.org/10.4000/remi.968

Ce document a été généré automatiquement le 14 avril 2022

(c) Université de Poitiers 


\section{Les camps d'étrangers depuis 1938 : continuité et adaptations}

Du « modèle » français à la construction de l'espace Schengen

Olivier Clochard, Yvan Gastaut et Ralph Schor

1 Selon Marc Bloch, « on comprendra toujours mieux un fait humain, quel qu'il soit, si on possède déjà l'intelligence d'autre fait de même sorte " (Bloch, 1959: 74). Certes les liens de parenté ne sont pas toujours évidents; mais en réinterrogeant cette notion de camp dans une perspective socio-historique, ne pouvons-nous pas dire que le camp de Sangatte, la zone d'attente de Roissy, le centre de rétention de Arenc (Marseille) ont un fonctionnement classique sous l'apparence d'une physionomie moderne? Lorsqu'un État maintient des personnes étrangères en quête d'une protection ou d'une vie meilleure dans des lieux d'enfermement, il établit une forme d'aliénation pour ces personnes qu'il ne veut pas voir ailleurs sur son territoire (Brunet, Ferras et Théry, 1992). La terminologie «camp de réfugiés » ou «camp de transit» renvoie de fait à l'idée d'un monde concentrationnaire, même si ces lieux d'exception ne sont pas toujours des espaces fermés entourés de barbelés ${ }^{1}$.

\section{Les camps des « années noires »}

2 Les camps d'internement en France n'ont pas manqué de retenir l'attention des historiens notamment pour la période de la Seconde Guerre mondiale. Passée une phase de silence tant dans l'opinion que chez les chercheurs, à partir des années soixante-dix, quelques études générales dont celle de Gilbert Badia, Les barbelés de l'exil (1979), accompagnées de monographies et d'une somme de témoignages publiés ont déjà abordé la question. Puis, avec les années quatre-vingt dix, la progression de la recherche dans ce domaine s'est amplifiée avec d'importants travaux tels ceux d'Anne Grynberg et de Denis Peschanski (Grynberg, 1991; Agamben, 1995; Brossat, 1997 ; Razac, 2000 ; Peschanski, 2002a).

3 La période des "années noires " délimitée par les analyses de François Bédarida et Jean-Pierre Azéma entre 1938 et 1948 (Azéma et Bédarida éds, 1993), représente en 
matière d'exclusion de l'Autre, un moment complexe et fondateur: les camps ont représenté une expression spatiale du rejet. Isoler pour mieux contenir et surveiller, séparer les populations concernées du reste de la société : telle était la logique du « regroupement» facilitant la tâche d'une administration soucieuse de maîtriser une population massive « en attente » ou « en transit $»^{2}$.

4 L'apparition de camps a résulté de situations extraordinaires, conséquences de dictatures militaires, guerres civiles, contextes économiques ou sanitaires difficiles. Pourtant sous la III ${ }^{\text {ème }}$ République, la naissance des camps ne revêtait pas un caractère d'urgence, pas plus qu'après la guerre en 1946. Les populations victimes, placées dans un statut d'exception, ont vécu des situations floues, aux bases juridiques inexistantes voire niées.

5 La multiplication de ces lieux inaugura une pratique qui s'est pérennisée après la guerre, même si les contextes, les conditions et les objectifs étaient sensiblement différents. En matière de rejet de l'immigré, les camps d'internement puis de rétention attestent d'une continuité : le lieu reste, les populations exclues passent en fonction des conjonctures politiques et économiques.

6 La séquence historique de la Seconde Guerre mondiale, de son amorce en 1937-38 à son épilogue en 1948-49, présente un intérêt majeur pour l'étude des mécanismes du rejet. $\mathrm{Au}$ carrefour de l'antisémitisme, du rejet des travailleurs immigrés, de l'inhospitalité à l'égard des réfugiés et apatrides, cette décennie, dont l'épicentre est représenté par l'idéologie de Vichy, met en scène différentes formes de discrimination dont les camps sont la plus visible des concrétisations. Le passage de la paix à la guerre puis le retour à la normalité n'ont pas altéré l'utilité des camps: à chaque situation, l'internement collectif de populations est apparu comme une nécessité.

\section{Un aboutissement de la politique d'immigration de l'Entre-deux- guerres}

7 Les camps d'internement sont contemporains de la mise en place d'une politique d'immigration. Preuves d'une gestion brutale et peu humaine des mouvements de population, ils répondaient à une volonté affirmée des pouvoirs publics depuis le début des années trente. Il s'agissait, à la suite de la loi restrictive de 1932, de maîtriser les entrées d'étrangers dans l'Hexagone tout en cédant à une xénophobie en forte progression au sein de l'opinion publique, analysée en profondeur par les travaux de Ralph Schor (1985).

8 Si les gouvernements de Front populaire entre 1936 et 1937 avaient choisi de rompre avec les politiques restrictives en matière d'immigration, l'arrivée à la Présidence du Conseil des radicaux Camille Chautemps entre juin 1937 et mars 1938 et surtout d'Édouard Daladier à parti d'avril $1938^{3}$ marqua un nouveau durcissement de la législation. Dès le 14 avril, le ministre de l'Intérieur Albert Sarraut demandait une action méthodique, énergique et prompte en vue de « débarrasser notre pays des éléments indésirables trop nombreux qui y circulent et y agissent au mépris des lois et des règlements ou qui interviennent de façon inadmissible dans les querelles ou les conflits politiques qui ne regardent que nous ». Il faut dire que l'année 1937 avait été marquée par une quinzaine d'attentats politiques mettant en cause des étrangers. 
9 Dans ce contexte, les gouvernements envisagèrent de structurer sous la forme d'une politique d'immigration ce qui jusqu'ici n'était resté qu'une somme d'actions désordonnées. Preuve de cette volonté, Philippe Serre 4 fut nommé pour quelques mois, sous-secrétaire d'État chargé des services de l'immigration et des étrangers dans le quatrième gouvernement Chautemps 5 . Pourtant, l'accueil des réfugiés politiques n'était pas encore complètement remis en cause : un décret-loi du 2 mai 1938 réaffirmait le principe d'une protection particulière au réfugié (Weil, 1991).

10 L'origine officielle des camps en France remonte au mois de novembre 1938 lorsque le gouvernement, dans le cadre de ses orientations restrictives, promulgua une loi sur les "étrangers indésirables » : toute personne de nationalité étrangère soupçonnée de porter atteinte à la sécurité du pays pouvait désormais être détenue dans des «centres d'internement» de "rassemblement» ou "centres spécialisés» en raison de leurs antécédents judiciaires et de leur activité jugée «trop dangereuse pour la sécurité nationale». Deux mois plus tard, en janvier 1939, le premier camp français s'ouvrit effectivement à Rieucros sur un terrain montagneux près de Mende. Composé de quatorze bâtiments dont dix baraquements en bois, il était placé sous l'autorité du préfet de Lozère et surveillé par la gendarmerie locale. Il s'agissait d'une première tentative d'instaurer l'enfermement d'étrangers expulsés mais ne pouvant quitter immédiatement le territoire : les apatrides. Cette catégorie administrative concernait les personnes qui n'avaient pas officiellement de pays d'origine, un véritable casse-tête pour les pouvoirs publics. Ni le droit de rester, ni la possibilité de partir : le camp de Rieucros avait pour fonction initiale de gérer ces "expulsés inexpulsables» (Schaul, 1987). La naissance du camp ne s'est pas produite dans une conjoncture exceptionnelle, son statut juridique initial était compatible avec le fonctionnement régulier du régime républicain.

11 Les camps d'internement n'étaient pas forcément considérés à la base comme une mauvaise solution, ni comme une mesure raciste : dans l'esprit des hommes politiques ou des hauts fonctionnaires ainsi que dans l'opinion, ils étaient plutôt perçus comme une solution alternative, la réponse provisoire à une urgence. Même chez les militants, plusieurs témoignages ont attesté que les camps étaient perçus plutôt positivement au tout début, dans la mesure où, malgré le cantonnement dans un lieu clos, ils assuraient un minimum vital non négligeable : lit, nourriture, protection.

12 En novembre 1939, la politique d'internement alla encore plus loin, dépassant même le clivage Français-étrangers. Elle fut étendue aux citoyens français par une loi d'exception qui répondait aux troubles de la «drôle de guerre ». Désormais les préfets ne sanctionnaient plus seulement les délits officiellement commis, mais aussi ceux qui étaient susceptibles de l'être.

13 La logique des pouvoirs publics avait consisté d'une part à proposer une gestion pragmatique des réalités, face à un flux massif de population et d'autre part à isoler les étrangers ainsi que des Français, considérés comme une menace pour l'ordre public. Même si la III ${ }^{\mathrm{ème}}$ République n'a pas fait des camps un objectif prioritaire, le principe de regrouper pour mieux surveiller et punir était dans l'air du temps: "l'internement républicain » était lancé (Fisher, 2003). 


\section{Les réfugiés espagnols, premiers « regroupés » en 1939} orientales s'avérèrent insuffisants pour organiser les arrivées et maintenir l'ordre. Le 31 janvier, quatorze régiments militaires prélevés dans tout l'Hexagone furent mobilisés pour récupérer les arrivants et les acheminer vers des lieux de rassemblement. En quelques jours des camps furent construits à la hâte comme celui d'Argelès-sur-mer, considéré comme un «camp de concentration » selon la terminologie employée par l'Administration et notamment le ministre de l'Intérieur Albert Sarraut (Rubio, 1994). Deux autres camps furent aménagés à proximité au milieu du mois de février à Saint-Cyprien et Barcarès. Puis de nombreux autres apparurent, tel celui du Vernet d'Ariège ou celui de Gurs construit en six semaines entre mars et avril 1939.

17 Selon Denis Peschanski, la grande majorité de ces réfugiés espagnols - environ 350000 - ont séjourné dans les camps (Peschanski, 2002a). À la suite des militaires, des groupements civils comme la Croix Rouge ou des élus mobilisèrent leur énergie. Mais les actes de solidarité restaient limités: entre une opinion française, indifférente, parfois hostile et des pouvoirs publics inactifs (Schor, 1985), les réfugiés espagnols souffraient des dures conditions de vie dans les baraquements sommaires, aggravées encore par le sentiment d'exclusion.

Dans les mois qui suivirent, les retours en Espagne furent nombreux. D'autres sortis des camps s'installèrent en France, refusant la dictature de Franco. Cependant, il restait encore de 120000 à 130000 internés au printemps 1940. L'exil temporaire se transforma dans certains cas en un enfermement de plus d'une année dans les camps. Le tri des personnes provoqua la séparation de familles, la promiscuité suscita des problèmes sanitaires et parfois des actes de violence. Le traitement fut encore plus 
sévère pour les militants révolutionnaires les plus actifs, considérés comme indésirables ou dangereux: certains furent transférés dans des camps éloignés, en Tunisie ou en Algérie.

19 De nombreux témoignages ont permis de conserver la mémoire des camps pour Espagnols à l'image de l'ouvrage de Geneviève Dreyfus-Armand et d'Émile Temime, Les Camps sur la plage, un exil espagnol (1995). Période certes limitée dans le temps, puisqu'en 1940, il n'y avait plus d'Espagnols internés, mais période de souffrance et de désillusions: l'expérience des camps français a marqué durablement toute une génération d'Espagnols. Sur le demi-million de réfugiés parti d'Espagne, seul un quart put y retourner : la plupart s'installèrent en France pendant et après la guerre après avoir combattu dans la Résistance.

\section{Au service des logiques racistes du gouvernement de Vichy et l'occupant allemand}

La politique des camps mise en place sous la III ${ }^{\text {ème }}$ République au coup par coup pour faire face à une conjoncture exceptionnelle fut installée dans la durée par le régime du maréchal Pétain.

21 Pendant la "drôle de guerre », déjà, d'autres catégories de personnes avaient rejoint puis remplacé les Espagnols dans les camps. À la suite de la déclaration de guerre, le 3 septembre 1939, un communiqué officiel avait en effet appelé les hommes adultes allemands et autrichiens à se rendre dans ces «centres de rassemblement ». Puis, en mai 1940, dans le contexte de l'offensive de l'Allemagne, un nouveau décret imposa l'internement des hommes et femmes ressortissants de " nations ennemies » venus de l'Europe sous domination nazie. Ces réfugiés juifs ou opposants à Hitler, furent internés à Rieucros, le plus répressif, à Gurs (Laharie, 1993 ; Schramm et Vormeier 1979) ${ }^{6}$, le Vernet, Bram, Saint-Cyprien, Les Milles près d'Aix-en-Provence ou encore Rivesaltes ${ }^{7}$, Récébédou à quelques kilomètres de Toulouse, Nexon en Haute-Vienne... Sousalimentation, manque d'hygiène, baraquements surpeuplés : la vie quotidienne était plus difficile que pour leurs prédécesseurs espagnols. Méprisés, soupçonnés d'espionnage, de trahison, l'opinion française n'était guère émue par ces mesures d'isolement qui semblaient justifiées.

22 Avec l'Occupation, la situation s'aggrava plus encore: victimes de rafles, de déportations, la plupart d'entre eux mourut dans les camps de concentration. Anne Grynberg dans Les camps de la honte (1991) a proposé une étude précise sur les conditions de vie des Juifs internés dans les camps français qui avaient servi à regrouper les Espagnols quelques mois plus tôt, insistant sur le système hiérarchisé des camps de manière verticale, du ministre de l'Intérieur au gardien.

Le gouvernement de Vichy ajouta à la législation répressive une série de lois promulguées entre l'été et l'automne 1940 dont le but était d'exclure de la société française avec plus de fermeté encore, les individus considérés comme dangereux pour la nation et la sécurité publique. Les Juifs furent les principaux visés : en octobre, un décret-loi donnait pour mission aux préfets d'interner ceux qui n'avaient pas la nationalité française. Entre 1940 et 1942, le gouvernement de Vichy privilégia ce mode d'exclusion davantage que l'occupant allemand investi dans d'autres tâches et d'autres pays : l'administration des camps était française la plupart du temps en Zone sud comme en Zone nord . Une inspection générale des camps dépendante du 
gouvernement, fut créée en 1941 mais sans guère de moyens. Le seul internement ordonné par les Allemands en octobre 1940 concernait les populations tsiganes. Sans avoir été déportés, ni exterminés, la majorité des tsiganes - entre 6000 et 7000 - ont passé toute la guerre dans des camps, soumis à une «assignation à résidence » dans des conditions de vie quotidienne déplorables (Peschanski, Hubert, Philippon, 1994 ; Filhol, 2001).

En revanche l'internement des Juifs, décidé par les dirigeants nazis au cours de l'année 1941, s'inscrivit dans une autre dimension dont le gouvernement de Vichy n'avait pas la maîtrise. Les premières rafles furent ordonnées par les Allemands à Paris en mai 1941 : 6500 Juifs polonais, tchécoslovaques, ex-autrichiens furent regroupés dans des camps de la région parisienne de Pithiviers ou de Beaune-la-Rolande. Puis en août, 4200 autres Juifs étrangers mais aussi français furent rassemblés dans le nouveau camp de Drancy gardé par une police française brutale et sévère (Schor, 1996).

Si au départ l'objectif avait été de prévenir un éventuel complot judéo-bolchevique, par la suite, il s'agissait de contribuer à la "solution finale », projet d'anéantissement total de la population juive, décidée lors de la conférence de Vannsee le 20 janvier 1942. À partir du printemps de cette même année, certains des camps français allaient servir de lieu de transit vers les « camps de la mort ». Les Allemands profitèrent de l'existence de ces infrastructures pour ordonner regroupement et déportation des populations avec la collaboration active des autorités de Vichy9. Les rafles du Vel D'Hiv, effectuées les 16 et 17 juillet 1942, marquent un moment d'apogée du racisme des " années noires » : elles aboutirent à l'internement de près de 13000 Juifs pour la plupart à Drancy qui servit de camp de transit avant leur déportation vers les camps de concentration ou d'extermination en Europe centrale.

Organisées par le président du Conseil, Pierre Laval et son secrétaire général à la police René Bousquet ${ }^{10}$, les rafles se poursuivirent jusqu'en 1944. Appliquant les directives allemandes, les pouvoirs publics français se chargèrent de gérer la déportation des Juifs de France dans l'espoir d'obtenir une place dans une Europe sous domination nazie. Pendant cette période, la Haute Administration française n'avait de cesse d'atteindre l'objectif fixé : rafler et regrouper un maximum de Juifs. Délaissés aux premiers temps de l'Occupation, les camps étaient devenus une pièce essentielle dans la stratégie raciste allemande, bien au-delà de la politique d'exclusion du gouvernement de Vichy. Selon Denis Peschanski, près de 100000 Juifs séjournèrent dans les camps français auxquels s'ajoutèrent 15000 opposants politiques français. Le gouvernement de Vichy n'interna pas seulement des étrangers: des individus de nationalité française notamment les communistes ou les francs-maçons subirent le même sort.

Les décès dans les camps français entre 1940 et 1944, dont le nombre a été établi à environ 3000 , était essentiellement dus à des maladies et surtout à une sousalimentation, signes d'une réalité tragique. La situation était tellement grave sur le plan humanitaire que des oeuvres d'entraide se mobilisèrent pour apaiser les souffrances et améliorer la prise en charge du quotidien. Un réseau de solidarité aux populations des camps rassembla de nombreuses associations : la Croix-rouge, l'Action catholique, la Fédération protestante de France, la Fédération des sociétés juives de France, l'HICEM (organisme juif d'aide à l'émigration), l'ORT (Société pour le développement du travail), l'OSE (Organisation du Secours à l'Enfance) ou la Cimade. Certaines d'entre elles, proches de la Résistance, organisèrent des évasions ou des passages clandestins de la zone occupée en zone libre. 

volonté de quelques militants ${ }^{11}$ dont la mission initiale était d'agir en faveur des "évacués» de l'Alsace Lorraine fuyant l'avancée nazie. Ses premières actions concernaient les camps : profitant de certains errements de l'administration, elle put pénétrer à Gurs. Dans un ouvrage intitulé Les clandestins de Dieu, une militante, Jeanne Merle d'Aubigné décrit ce camp comme une «mer de baraques sur $3 \mathrm{~km}$ de long et 1,5 km de large. L'ensemble était divisé en 12 îlots entourés chacun d'épais barbelés. A chaque porte d'îlot, un garde armé. 40 à 60 internés par baraque » (Cimade, 1985). À partir de fin 1940, la présence de militantes de la Cimade à Gurs devint permanente: installées dans des baraquements, elles apportaient une assistance matérielle, religieuse et une aide à la vie culturelle. génocide des nazis.

\section{Les camps de la Libération, autre forme de rejet}

La fin du régime de Vichy ne signifia pas la fin de l'histoire des camps : à partir de la Libération, ils eurent d'autres usages.

31

Une nouvelle politique d'internement fut en effet élaborée par le Gouvernement provisoire de la République française (GPRF). Il s'agissait d'une nouvelle conjoncture d'exception dans le cadre de l'épuration et de mesures de précaution pour la sécurité du pays. En octobre 1944, on comptait environ 60000 internés sur le territoire libéré, répartis dans 170 camps (Peschanski, 2002a). Par la suite, ce nombre a rapidement diminué selon les orientations fixées par le ministre de l'Intérieur, Adrien Tixier : en avril 1945, il ne restait que 20000 individus internés, et en décembre, 13000 . On comptait en grande partie des civils allemands renvoyés de la zone des combats vers l'intérieur du pays, notamment en Alsace. Des Français accusés de collaboration et notamment des femmes soupçonnées de relations sexuelles avec l'ennemi peuplaient également les camps (Virgili, 2000), victimes d'une épuration sans ménagement. Le dernier interné sortit en mai 1946.

L'enfermement à la Libération a répondu à une situation d'urgence et d'incertitudes qui ne correspondait ni à la logique de restriction des gouvernements de la III ème République, ni à la logique xénophobe du gouvernement de Vichy, ni à la logique d'extermination à l'encontre des Juifs prônée par les autorités allemandes. La même infrastructure représentée par le camp d'internement a été donc utilisée pour des politiques et des stratégies d'exclusion bien différentes. Denis Peschanski (2002a) propose quatre logiques successives des camps entre 1938 et 1946: une logique républicaine entre novembre 1938 et juin 1940, une logique vichyssoise entre juin 1940 et l'été 1942, une logique nazie, vers l'extermination entre l'été 1942 et l'été 1944 et une logique de la Libération entre l'été 1944et mai 1946.

Les tensions de l'après guerre en voie d'apaisement, l'opinion envisagea le rapport aux étrangers avec plus de sérénité, d'autant que la conjoncture nécessitait un appel de la main d'œuvre étrangère. À la Libération, les pouvoirs publics s'engagèrent dans l'élaboration d'une politique d'immigration qui se voulait dirigiste, structurée et efficace. Les différentes formes de rejet du temps des années d'Occupation n'étaient plus de mise. Pourtant, le recours aux camps ne fut pas exclu. En 1946 et 1947, dans le cadre de la gestion de l'immigration, le ministre du Travail, Ambroise Croizat prit des

Revue européenne des migrations internationales, vol. 20 - n² | 2004 
mesures énergiques pour contrôler les flux vers la France : refoulements à la frontière et « regroupement » des clandestins interpellés.

Dans les Alpes-Maritimes, par exemple, l'important flux d'Italiens vers la France provoqua une inquiétude de l'opinion locale et des pouvoirs publics. Plusieurs circulaires du préfet ${ }^{12}$ ordonnèrent la création d'un camp pour les immigrés italiens clandestins, construit à la hâte à proximité de la frontière à Menton ${ }^{13}$. Des voix s'élevèrent contre ces mesures, à l'image d'un délégué local de la CGT parlant d'un "véritable camp de concentration qui donne une bien mauvaise image de la démocratie française $»^{14}$. Il est particulièrement intéressant de constater que lorsque le danger d'une "invasion » de réfugiés fut éloigné, ce camp de Menton se transforma en une succursale de l'office national d'immigration (ONI) créé en 1946 pour permettre la régulation des entrées d'immigrés en France. En France comme à l'étranger, les différents centres de recrutement de l'ONI correspondaient à d'autres formes de regroupement, moins exceptionnelles, pour fournir une main-d'œuvre saine et solide à l'économie française.

Les camps d'internement sont donc apparus en France à la charnière entre Troisième République et "Révolution nationale ». Peur et haine de l'Étranger agitaient si fortement les esprits à ce moment crucial que le principe des camps, hérité du commode casernement militaire afin d'éviter la dispersion et surtout le mélange, fut adopté. Bien sûr, la thèse établissant une filiation entre une République qui engendra les premiers camps d'internement et le régime de Vichy qui les utilisa pour mettre en place un antisémitisme d'État est largement contestable. Mais les mesures répressives élaborées dans les années trente contre les étrangers ont influencé et dans une certaine mesure conditionné l'opinion française habituée à confondre travailleurs immigrés, réfugiés et Juifs. L'amalgame était facile entre altérité et « fauteurs de guerre » d'autant plus que la période était particulièrement troublée. Pierre Laborie (1992) ou Denis Peschanski (2001) ont démontré un comportement xénophobe généralisé tant dans les institutions que dans l'opinion au cours de la période des " années noires».

Ce constat autorise à envisager une continuité dans l'utilisation du camp comme moyen de rétention et d'internement temporaire de populations jugées indésirables depuis l'entre-deux-guerres. Comme sous la III ème République, sous la IV ème et sous la V ème République, bien que les enjeux et les formes aient été totalement différents, les phénomènes d'exclusion ont été mis en scène à travers le cloisonnement spatial. Un colloque organisé en 1995 sur le thème Camps de concentration, un phénomène du XXème siècle ${ }^{15}$ a bien montré ces permanences à une échelle plus large avec en toile de fond les écrits d'Hannah Arendt sur le système concentrationnaire.

L'internement constitue un phénomène complexe dont l'histoire et la mémoire peinent à rendre compte, notamment à cause de la diversité des situations, des populations concernées et des enjeux souvent enchevêtrés. La question des camps éclaire ainsi une superposition de « racismes français » : un rejet des juifs, le plus violent, augmenté d'un rejet des travailleurs immigrés, augmenté encore d'un rejet des réfugiés à l'image des Espagnols ou apatrides comme les Tsiganes. Ces formes de rejet trouveront leur expression dans les camps, véritables lieux d'exclusion. 


\section{Les camps aujourd'hui} à vivre au premier étage du terminal «ferries ». Les étrangers étaient allongés en rang d'oignons, soit pour dormir, soit pour attendre durant la journée une éventuelle solution à leur situation. Face à cette "gêne grandissante ", les autorités locales apportèrent à plusieurs reprises une aide matérielle à ces exilés dans un hangar, une salle désaffectée de l'hôpital... À ces structures d'accueil caractérisées par l'urgence et une brève durée d'existence, succédaient dans divers espaces de la ville des camps éphémères montés de bric et de broc par les étrangers en transit à Calais. Cette crispation atteint son paroxysme au cours de l'été 1999. Le Parc Saint-Pierre, face à la mairie, offre progressivement l'image d'un bidonville : les gens édifient des installations de fortunes avec des films plastiques ou des sacs poubelles tenus par des matériaux de récupération, des plaques de polystyrènes ou de placoplâtre "empruntées " sur un chantier voisin, le tout assemblé avec du fil de fer. Non loin du port, sur un terrain vague, d'autres "réfugiés" cherchent aussi à s'abriter et établissent un autre campement dissimulé par quelques fourrés. Dans ce deuxième campement, les constructions rassemblent une trentaine de cabanes qui ne cessent de se consolider au fil des semaines. Des femmes enceintes, d'autres avec des bébés vivent dans ces abris de fortunes avec comme matelas des cartons ou des plaques de laines de verre recouvertes de tissu. Lors des intempéries, les "réfugiés " s'orientent vers d'autres lieux dont l'accès se limitait généralement à une nuit: un bâtiment en construction, des logements désaffectés, des blockhaus, la gare SNCF voire des wagons stationnés en gare de Calais.

40 À la fin de l'été 1999, le gouvernement réquisitionne l'ancienne usine à voussoirs d'Eurotunnel qui deviendra le camp de Sangatte. Si ce lieu vise à apporter une aide humanitaire aux "réfugiés » errant dans Calais et ses alentours, il introduit dans le même temps un effet dissuasif auprès de ces exilés qui souhaiteraient rester en France. Certes un grand nombre d'entre eux projette de se rendre en Angleterre, mais les conditions d'accueil et l'information au compte goutte sur le droit d'asile découragent ceux qui envisagent de solliciter une protection auprès de la France. En mobilisant les services de la Croix Rouge et d'importants effectifs policiers, l'État tente d'assurer, en quelque sorte, la garde de ces indésirables, hors de la vue des habitants. Mais au fil des années, l'augmentation du nombre des « réfugiés » dans le camp rend à nouveau visible leur concentration dans la région où ils étaient tolérés. La fermeture du camp en 
décembre 2002, réclamée depuis longtemps par les autorités anglaises, conduit à la dispersion des étrangers le long de la frontière maritime de l'espace Schengen (notamment dans les ports) qui fait face au Royaume-Uni.

41 Pour comprendre cette situation de crise, il semble important de situer cet événement dans un cadre spatio-temporel plus large. En effet, l'exemple du camp de Sangatte et du Calaisis présenté au départ comme une exception s'inscrit tout compte fait dans une problématique européenne. La plupart des pays européens adoptent des dispositifs visant à mettre à l'écart les étrangers démunis de papiers en règle. Une grande partie de ces nasses aux frontières de l'Union européenne, de part leur localisation, ne sontelles pas les prémices à l'externalisation du droit d'asile qui se dessine dans divers projets européens?

\section{Des camps d'étrangers à l'euphémisme des termes désignant les lieux d'enfermements}

À travers l'exemple du centre d'hébergement et d'accueil d'urgence humanitaire (CHAUH) de Sangatte, cette figure de rhétorique par laquelle les autorités ont tenté de voiler l'accueil mérite de s'y arrêter quelques instants. Le premier rapport de mission effectué sur le camp de Sangatte par un groupement d'associations ${ }^{16}$, a employé le terme de "camp» pour souligner les conditions d'accueil des étrangers sous cet immense bâtiment de tôles ; le rapport a choqué la direction de la Croix Rouge et les pouvoirs publics qui ont toujours refusé de désigner le hangar d'Eurotunnel où vivaient des personnes comme un camp. En juillet 2001, lors d'un entretien avec un responsable de la Croix Rouge à Sangatte, lorsque le mot «camp » est évoqué, ce dernier déclare "qu'il vaut mieux dire centre, parce que "camp" renvoie à de mauvais souvenirs"; toutefois son opposition n'est plus aussi formelle lorsque la remarque est faite dans l'espace où sont dressées les tentes (carte ci-dessous). Plusieurs auteurs ont aussi réfuté l'idée de camp de réfugiés ou de camp d'internement ${ }^{17}$; par ailleurs, Luc Legoux précise que pour l'un de ses articles (2002), l'équipe de rédaction des Cahiers Français revue publié par la Documentation Française, s'est toujours opposée à l'emploi du terme "camp " pour désigner ce lieu. Le camp de Sangatte n'en était pas moins une des configurations de mise à l'écart des étrangers en France. Même si lors des intempéries, les "réfugiés " de Calais n'ont pas eu à creuser avec leurs mains des trous dans le sable pour s'abriter $\mathrm{du}$ froid, comme les républicains espagnols, la période de «l'après Sangatte » montre encore des conditions parfois inhumaines auxquelles sont confrontés des étrangers en quête d'une vie meilleure. Les diverses structures qui ont marqué "l'accueil d'urgence » dans la région de Calais, sont bien la marque du provisoire, expression empruntée par Émile Témime lorsqu'il parle des camps sur la plage. "Ce n'est parfois que l'affaire de quelques heures; et, parfois, cette hésitation peut se prolonger des semaines, des mois, des années. Séjours imposés (la "quarantaine", le camp de "regroupement", voire le centre de "rétention"...), qui traduisent toujours une méfiance, et parfois un rejet. Résidences éphémères, chambres d'hôtel où l'on ne fait que passer, dans l'attente, dans l'espérance d'un nouveau départ. Ce sont souvent des lieux de misère, qui se rattachent à l'histoire même de l'immigration et de l'exil, et dont on gommera volontiers le souvenir gênant" (Milza et Témime, $2001: 5$ ). Aujourd'hui, seule une grande dalle de béton rappelle la présence du camp de Sangatte, le hangar fut détruit dans les semaines qui suivirent la fermeture officielle. 
En Europe, l'ensemble des lieux spécifiques au maintien des étrangers s'inscrit dans ce processus (carte). En Italie, les Centri di permanenza temporanea e assistenza (centres de permanence temporaire et assistance) sont de temps en temps encore nommés sous l'ancienne terminologie officielle toute aussi euphémique: «centres d'accueil». En Belgique, les six principaux lieux de détention sont appelés "centres fermés ». Sur l'enceinte ou à proximité de l'aéroport de Bruxelles, les deux lieux qui hébergent des étrangers arrivant sur le territoire belge, sans document de voyage valide, sont le centre INAD (pour INADmissibles) à Zaventem ou le centre 127 bis à Steenokkerzeel. Dans le principe, la numérotation du dernier lieu renvoie à l'anonymat le plus total. En Allemagne, des «centres de départ volontaire » ont été mis en place pour inciter des étrangers, démunis de carte de séjour ou déboutés de leur demande d'asile, à quitter le pays. En Angleterre, l'ancienne prison de Campsfield House, pour jeunes délinquants, a été réhabilitée en 1993 pour devenir un des plus grands centres de rétention d'Angleterre. À son ouverture, le gouvernement le désignait comme un "foyer sûr »; mais en 1995, un rapport gouvernemental avouait que "sur place personne ne pouvait établir clairement la différence $»^{18}$. À Ceuta, le "Centro de Estancia Temporal para Inmigrantes » est souvent désigné par le sigle «CETI », à l'instar de la principale zone d'attente française "ZAPI» située en bordure des pistes de l'aéroport de Roissy. Or pour une partie des étrangers qui sont amenés à transiter par ces lieux, ces sas s'apparentent avant tout à des centres de refoulement, d'expulsion. "L'emploi adoucissant» de ces termes vise à diluer au sein des démocraties de l'Union européenne des réalités trop choquantes; comme si la volonté de ceux qui baptisent ainsi ces espaces, cherchaient à masquer leur irruption dans l'espace public ${ }^{19}$. Si la notion "camps d'étrangers" prête indubitablement à la discussion, en écartant l'euphémisation ambiante liée à ces espaces physiques, elle permet également de mieux montrer les diverses situations d'enfermements des étrangers au tournant du XX ${ }^{\text {ème }}$ et XXI ${ }^{\text {ème }}$ siècles.

\section{Visibilité et invisibilité de ces lieux d'enfermement}

Les formes de ces camps sont très diverses, ne correspondant pas toujours à l'image qu'évoque ce terme. Mais, quelle que soit leur apparente « ouverture », il s'agit de lieux d'enfermement. 


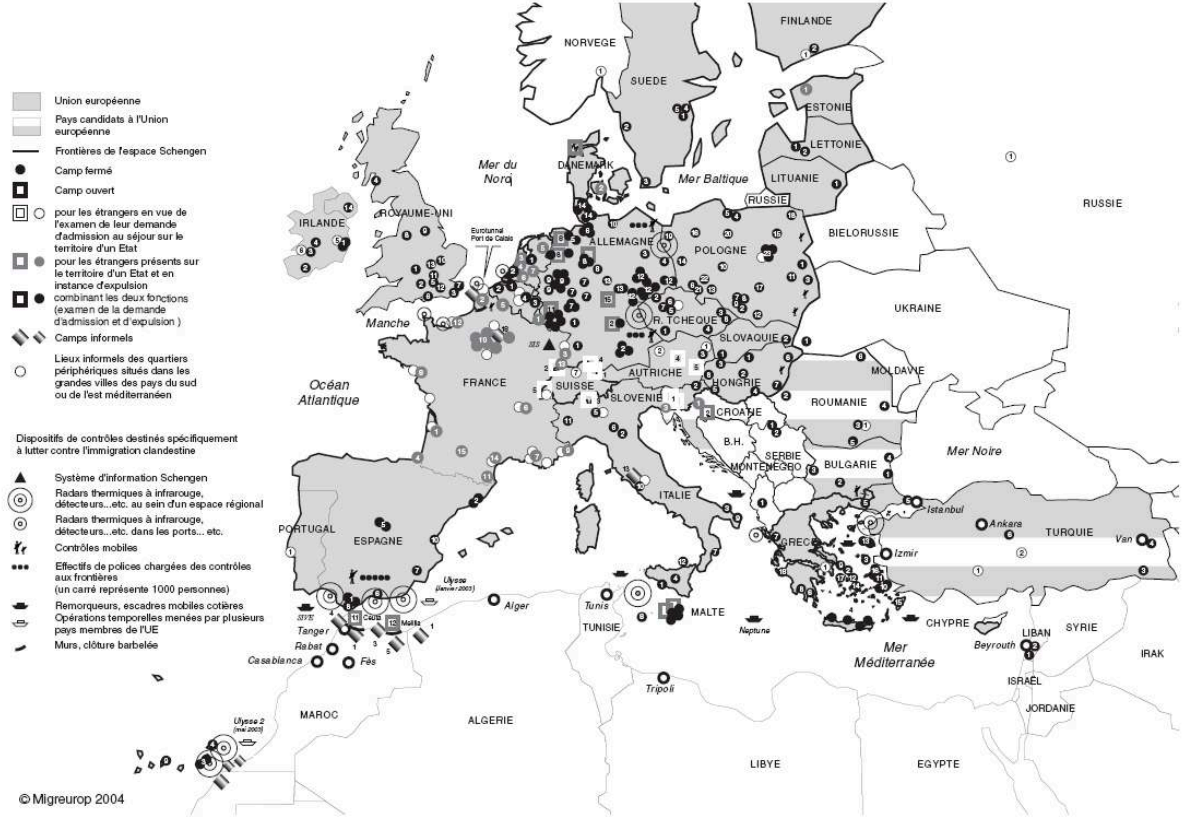


Camps d'étrangers en Europe et dans les pays méditerranéens. Note méthodologique

Si le réseau Migreurop propose une typologie relativement large de la notion de "camps ", face à l'hétérogénéité et à l'importance numérique de ces lieux à l'intérieur et aux frontières de l'espace de l'Union européenne, nous avons choisi pour l'élaboration de cette carte de représenter principalement les lieux physiques d'enfermements des étrangers où ils sont totalement privés de leur liberté de circulation (« camps fermés »).

Pour la France, sont cartographiées uniquement les zones d'attente qui reçoivent régulièrement des étrangers.

En Allemagne, sont représentées les prisons ayant une place importante dans le dispositif du maintien des étrangers en situation irrégulière.

En Suisse la détention d'étrangers en vue de leur refoulement a souvent lieu dans des sections spéciales d'établissements pénitentiaires ordinaires. La superficie de la Suisse permet difficilement de cartographier les 23 lieux connus: Appenzell, Bâle (2), Bern, Chur, Dornach, Einsiedeln, Gampelen, Glarus, Granges, Mendrisio, Olten, Saignelégier, Schaffhausen, Schüpfheim, Sissach, Solothurn, Sursee, Thônex, Widnau, Zug, Zürich (2).

Voir en annexe des précisions sur la légende ainsi que la liste des camps représentés

Sources: Allemagne: Initiativen gegen abschiebehaft-Berlin / Autriche: Asylkoordination Österreich / Belgique: Mrax / Danemark: http://www.coe.int/T/F/Com/Presse/Actualite/ Espagne: Rapport du Comité européen pour la prévention de la torture et des peines ou traitements inhumains ou dégradants (CPT) (6/8/2003) ; Luz Saavedra / Estonie : Legal information centre for human rights / France : ministère de l'Intérieur français / Grèce : Articles de presse (http://www.enet.gr/online/online) / Hongrie : Hungarian Helsinki Committee, helsinki@mail.datanet.hu / Irlande : Irish refugee council / Italie : Storie in gabbia, supplément à Il Manifesto du 31/5/03, http://www.migranti.net/pages/inserto_CPT.pdf / Lettonie : The latvian centre for human rights and ethnic studies / Lituanie : rapport de M. A. Gil-Robles, commissaire aux droits de l'Homme sur sa visite en Lituanie (2004) http://www.coe.int/T/f/commissaire_d.h/ Luxembourg: http://www.cpt.coe.int/documents/lux/2004-12-inf-fra.pdf / Malte: jrsmalta@waldonet.net.mt / Pays Bas : www.autonoomcentrum.nl / Pologne : JRS / République Tchèque : http://www.mvcr.cz/suz/uvod.html, Sandrine Carton (2003) «L'institutionnalisation de l'asile en Europe centrale: l'exemple tchèque. 1990-2003", Paris I / Royaume Uni : http:// www.barbedwirebritain.org.uk/ / Slovaquie: JRS / Slovénie : JRS / Suède: Migration Board, www.migrationsverket.se/english/maps and addresses / Suisse : Organisation suisse d'aide aux réfugiés, www.sfh-osar.ch, Office fédéral des réfugiés, Office fédéral de la statistique, Conférence des directrices et directeurs des départements cantonaux de justice et police / Gross Andrea (2000) Arrivée de demandeurs d'asile dans les aéroports européens, Conseil de l'Europe.

Bulgarie : Croix Rouge Bulgare, Bulgarian Helsinki Committee / Croatie: Croix Rouge croate, Croatian Law Centre / Roumanie : Bénédicte Michalon - Migrinter-CNRS / Serbie et Montenegro : Groupe 484, Gracanicka 10, Belgrade / Ukraine : Ukrainian State Committee

Algérie : Association "Rencontre et développement ", Alger / Liban : fidh@fidh.org / Maroc : AFVIC / Turquie : HCR, International Catholic Migration Commission, JF Pérouse - IFEA /

Migreurop ne dispose pas de donnée pour l'Egypte, l'Israël, la Libye, la Syrie et la Tunisie [pays situés au Sud et à l'Est de la Méditerranée] ainsi que pour la Biélorussie, la Bosnie-Herzégovine, la Macédoine, la Moldavie et le Monténégro. Concernant la Norvège, le Portugal et la Russie, seules les informations, issues du rapport européen d'Andréa Gross (cf. ci-dessus), sont cartographiées. 


\section{Le camp fermé}

45 géographiquement identifié, et dévolu au placement d'indésirables» (Migreurop, 2003). La majeure partie de ces lieux ont généralement des périmètres très marqués : enceintes murées, miradors, caméras, barbelés. Quand ces derniers n'existent pas, les clôtures grillagées surmontées de fils de fer barbelé « concertina » sont devenues les matériaux courants utilisés pour la délimitation de ces espaces frontaliers. Ces clôtures s'apparentent à " un trait qui barre l'espace et évoque immédiatement la privation de liberté (comme le feraient des barreaux de prison)» (Razac, 2000: 64). Elles rappellent constamment l'interdiction faite aux étrangers maintenus de circuler librement. $\mathrm{Au}$ sein de l'Union européenne, la mise à l'écart d'étrangers dans des lieux prévus à cet effet s'opère de manières très diverses. Dans les grands aéroports et ports, la rétention peut s'effectuer dans des salles, des hangars, des bâtiments dont la fonction initiale n'est pas destinée à l'enfermement; la fonction de ces micro espaces est ainsi noyée dans l'activité principale de l'infrastructure. Certaines de ces structures ne sont que temporaires, d'autres perdurent dans le temps.

En France, si les centres de rétention et les zones d'attente sont destinés spécifiquement au maintien des étrangers, les prisons et les postes de polices sont également utilisés (en 2001, 25 \% des étrangers incarcérés, soit 4295 personnes, ont été écroués pour avoir refusé de se soumettre à une mesure d'éloignement). L'Allemagne et l'Irlande se distinguent par l'enferment de nombreux étrangers dans des centres pénitenciers, à l'instar du Royaume-Uni qui possède des structures spécialement destinées aux étrangers démunis de titre de séjour. En République Tchèque, à Cerveny Ujezd, depuis le 26 juin 1991, l'ancienne base militaire de l'armée soviétique « reçoit à la fois des demandeurs d'asile en quarantaine et des réfugiés hébergés là en permanence " (Novak, 1994). Dans les îles de la mer Égée, les autorités grecques réquisitionnent, souvent dans l'urgence, des campings, des centres sportifs, des lieux désaffectés (hôtels, entrepôts, caserne, prison, ancien immeuble de journal). Enfin l'utilisation de plus en plus fréquente par les migrants de la route maritime entre les côtes libyennes et les îles italiennes, a conduit les autorités maltaises à cette logique d'enfermement; tout étranger arrivant illégalement sur l'île ou en situation irrégulière est enfermé, y compris les demandeurs d'asile.

$\mathrm{Si}$ «le barbelé [et les systèmes adjacents] a acquis ce pouvoir symbolique exclusif» (Razac, 2000 : 65) du camp, il semble important de ne pas occulter les autres configurations qui en résultent. Pierre Bourdieu indiquait que "l'espace est un des lieux où le pouvoir s'affirme et s'exerce, et sans doute sous la forme la plus subtile, celle de la violence symbolique comme violence inaperçue» (Bourdieu, 1993 : 163). En effet, les techniques de gestions des flux migratoires à l'aide de bâtiments faisant office d'hébergement ou sur des espaces ouverts engendrent des procédés analogues à celles des lieux clos, mais d'une manière beaucoup plus discrète, bien que toute aussi brutale.

\section{Le camp ouvert}

«La diversité des dispositifs administratifs et des contraintes technico-humanitaires visant à regrouper des migrants invite à dépasser la seule référence à l'enfermement et à considérer comme des camps l'ensemble des lieux de mise à distance des étrangers. Les formes que celle-ci 
peut prendre, sont multiples et parfois très éloignées de celle du "camp avec barbelés". Ainsi certains centres " ouverts » d'accueil, de transit ou d'hébergement ont une vocation apparente apporter une assistance et un toit - qui masque mal le fait que leurs occupants, migrants et demandeurs d'asile, ne disposent en général d'autre choix que de s'y trouver" (Migreurop, 2003). En Belgique ou au Danemark, les demandeurs d'asile sont contraints à vivre dans des centres d'accueil non choisis sous peine de ne pas bénéficier d'aide sociale (allocation financière et soutien à l'instruction de leur demande d'asile). Il en est de même en Allemagne; de plus les déplacements des requérants sont soumis à une restriction géographique.

49 Les camps ouverts de Melilla et Ceuta s'inscrivent dans cette classification, de même que le camp de Sangatte. Dans le Calaisis, jusqu'au début du mois de novembre 2002, certes les étrangers avaient la possibilité de circuler librement, mais la plupart des exilés présents dans la région étaient " conduits systématiquement» vers ce lieu, au gré des arrivées, afin de limiter leur présence dans les espaces publics de la ville de Calais. "Mise à l'écart physique et surtout mise à l'écart juridique : ces réfugiés, bien qu'ils soient tous notoirement en situation irrégulière et à la recherche d'un pays d'accueil, n'ont pas été poursuivis et très peu informés de la possibilité de déposer une demande d'asile en France " (Migreurop, 2003).

\section{Les lieux caractérisés par l'errance des étrangers}

50 "L'analyse des facteurs d'émergence des lieux où l'Europe isole certains étrangers invite, à une acception encore plus large de la notion de camp. L'errance des exilés qu'on disperse pour éviter la création de nouveaux abcès de fixation ne symbolise-t-elle pas le caractère multiforme que peut prendre le concept de mise à l'écart des étrangers par les sociétés européennes, sans qu'il soit besoin de barreaux ni de murs pour entraver leur circulation et leur légitime recherche d'une terre d'accueil? Ne peut-on assimiler à une informelle "assignation à résidence" l'obligation, pour des étrangers, de n'être pas à un endroit où ils sont considérés comme gênants? Le harcèlement policier et l'obligation d'invisibilité font alors office de barreaux et tracent les limites des lieux de relégation réservés aux étrangers" (Migreurop, 2003). Les épisodes antérieurs à l'ouverture du camp de Sangatte s'inscrivent dans ce processus de mise à l'écart : les jardins publics de Calais ont vu défiler de nombreux exilés; depuis la fermeture, ces lieux d'exil se recomposent en permanence dans la ville et sont apparus sous d'autre forme dans le quartier de la gare du Nord à Paris, notamment dans le square Alban-Satragne du X ${ }^{\text {ème }}$ arrondissement.

\section{Une grande diversité dans l'exercice de ces lieux}

51 Quelle que soit la catégorie à laquelle le lieu appartient, il ressort des enquêtes effectuées au sein du réseau Migreurop une grande hétérogénéité des camps à l'intérieur et aux frontières de l'Union européenne. Au sein de ces espaces, deux situations d'enfermement se présentent :

- - la personne souhaite entrer sur le territoire au titre de l'asile, pour un séjour lié à d'autres raisons ou en vue d'un simple transit afin de poursuivre son voyage vers un autre pays. Suite à l'examen de sa situation, l'étranger peut être admis immédiatement sur le territoire, ou être renvoyé vers sa destination de provenance si les autorités estiment qu'il ne présente pas les conditions nécessaires à son admission. Dans certains grands aéroports européens, les étrangers ne sont pas censés être sur le territoire de l'État. 
- la personne est démunie de titre de séjour lui permettant de rester sur le territoire de l'État. Les étrangers déboutés de l'asile et/ou du droit au séjour sont alors sous le coup d'une mesure d'expulsion.

52 Toutefois la plupart des lieux en Europe combinent les deux fonctions. Seuls la Suisse, les Pays-Bas et la France semblent faire clairement la distinction. Pour rappel, en France, les centres de rétention servent à l'éloignement des étrangers " sans-papiers » et les zones d'attente sont destinées à empêcher l'entrée bien que le spectre de l'expulsion y soit très présent. Toutes ces formes de mise à l'écart, gérées par des organismes humanitaires, des sociétés privées (comme en Italie ou en Angleterre), des policiers ou des militaires ont des fonctionnements très différents de par leur cadre juridique, la durée du séjour et la dépersonnalisation à laquelle les exilés sont parfois confrontés.

\section{L'environnement juridique}

53 Ne pas avoir de papier d'identité, de document de voyage en règle n'est pas un délit, c'est avant tout une difficulté administrative à laquelle beaucoup d'étrangers en quête de protection sont confrontés. Il n'existe pas de forme légitime de la détention pour les demandeurs d'asile. L'article 31 de la convention de Genève prévoit que les pays signataires ne peuvent appliquer aux réfugiés « de sanctions pénales, du fait de leur entrée ou de leur séjour irrégulier » et de "restrictions [quant à leur déplacement] que celles qui sont nécessaires». Depuis la fin des années quatre-vingt les États maintiennent cependant des étrangers dans des zones de transit ${ }^{20}$, au-delà des limites du "nécessaire " et parfois en dehors de tout cadre légal. Les autorités justifient ces créations comme un état d'exception à l'instar de la découverte en 1975 dans le port de Marseille, du centre de rétention d'Arenc. Au sein de grands aéroports européens (comme Rome ou Paris), le rapport de Lord Mackie of Benshie, rendu public le 20 juin 1991, précise que les procédures ne sont pas claires pour les agents de contrôle et aucune règle bien définie n'est appliquée. Si, en 1999, le rapport européen d'Andréas Gross note une amélioration au sujet du traitement des demandes d'asile à la frontière, aujourd'hui à Roissy, la rapidité des procédures rend l'accès en grande partie illusoire. Entre les droits accordés aux étrangers qui ne sont pas toujours appliqués et les politiques de lutte contre l'immigration illégale qui se traduisent par des renvois immédiats pour ne pas laisser accès aux procédures, les agents de contrôles aux frontières sont cernés par ces deux environnements ; ce qui engendre inévitablement une application de règles arbitraires.

54 Aujourd'hui encore dans plusieurs États de l'Union, des lieux dévolus au maintien des étrangers ne sont pas encadrés juridiquement. L'existence singulière de Sangatte entre octobre 1999 et décembre 2002, en marge de la législation réglementant l'entrée et le séjour des étrangers en France, est un exemple notoire.

\section{Les durées de détention très diverses}

55 La durée de détention dans ces lieux qui peuvent être considérés comme des nasses, varie considérablement et peut même être très longue. Au Royaume-Uni, quelle que soit la situation du demandeur d'asile, la loi sur l'immigration de 1971 laisse les requérants passible d'un séjour en rétention pour une période indéterminée; le postulat généralement admis au début des années quatre-vingt dix, d'un séjour ne 
dépassant pas trente à quarante jours, "devint vite obsolète avec la promulgation de Asylum and Immigration Appeals Act 1993, qui allongea la durée de rétention puisqu'il fallait attendre les décisions et le résultat des recours " (Harrel-Bondi et Popondo, 1996). Sur l'île de Malte, certains requérants auraient été internés 18 mois dans le centre fermé de Floriana $^{21}$; en République Tchèque, dans les centres de détention dont la plupart ont été mis en place en 1998, la durée maximale est de 180 jours. En Belgique, les étrangers peuvent attendre jusqu'à 5 ou 8 mois, selon si l'étranger s'oppose ou non aux forces de l'ordre, voire plus si le compteur est remis à zéro! En Espagne, si la détention ne dépasse pas 40 jours, les conditions sanitaires de certains lieux sont bien plus dégradantes que dans le milieu carcéral. À l'aéroport de Francfort-sur-le-Main, la décision sur la recevabilité de la demande d'asile est prise dans un délai de dix-neuf jours; et la durée moyenne du séjour est de huit jours bien qu'elle puisse atteindre trente jours. En France, avant la dernière réforme législative de l'ordonnance du 2 novembre 1945, le maintien de l'étranger en zone d'attente ne devait pas dépasser 20 jours selon la loi. À l'aéroport de Roissy, des exilés ont cependant vécu dans cet espace bien au-delà de cette durée. En effet lorsque ces derniers étaient refusés par les autorités de l'aéroport vers lequel ils avaient été expulsés (accompagnés ou non par les forces de l'ordre), les autorités de contrôle leur notifiaient à leur retour sur Paris un nouveau placement en zone d'attente. Depuis la dernière modification législative, la durée maximale de séjour dans les zones d'attente est passée de 20 à 24 jours et de 12 à 32 jours dans les centres de rétention. Quelle que soit la durée, ces mises à l'écart s'inscrivent dans la tradition de l'enfermement des étrangers au XX ${ }^{\text {ème }}$ siècle ; elles sont un moyen de dissuasion, de rappel à l'étranger qu'il est «l'Autre ", un requérant dont les intentions inspirent des doutes aux autorités.

\section{La dépersonnalisation}

56 Au sein de ces espaces, toutes les personnes sont passibles de la même peine : l'attente ou l'expulsion. Dans ces dispositifs, on ne connaît plus l'individu mais on gère le groupe. Pour exemple, au Tribunal de Grande Instance de Bobigny ${ }^{22}$, il arrive que des dossiers d'étrangers soient traités de façon collective du fait que ces derniers sont arrivés par le même vol. "Au début des années quatre-vingt dix, la police de l'air et des frontières avait "tatoué" à l'encre indélébile un groupe de Chinois maintenus à la zone d'attente de Roissy-Charles De Gaulle, alléguant que ces étrangers cherchaient à échapper aux poursuites en changeant volontairement leurs identités " (Blanchard et Rodier, 2003). Les patronymes des individus disparaissent au profit d'un groupe référencé soit à la nationalité, à l'ethnie, à la langue, à la classe d'âge, soit à la date d'arrivée ou des numéros. À Roissy, pour des raisons d'efficacité, les autorités agissent parfois rapidement lorsqu'il y a un grand nombre de maintenus. L'alias du passeport emprunté par l'étranger est maintenu sur le procès verbal bien que le demandeur d'asile mentionne son identité.

\section{L'externalisation du droit d'asile}

Les milliers d'étrangers qui sont internés dans ces camps européens sont de plus en plus considérés comme des "réfugiés économiques ", dans la perspective de la lutte à mener contre l'immigration clandestine. Depuis la fin des années quatre-vingt dix, avec la politique des accords de réadmission, la politique d'attribution des visas Schengen, les agents de liaison, le fichage biométrique, les patrouilles en mer Méditerranée..., les 
travaux de l'Union Européenne en matière d'immigration annoncent en quelques sortes la mise en place de l'idée du gouvernement britannique : créer des centres d'accueil pour demandeurs d'asile aux frontières extérieures de l'Union.

\section{Le projet britannique et le programme «Convention Plus » du HCR}

Danemark avait tenté de faire passer une proposition de ce type aux Nations Unies. En 1993, les Pays-Bas avaient à leur tour évoqué ce programme dans un plan intergouvernemental». Si cette proposition d'externaliser l'asile n'a pas été retenue par le Conseil européen de Thessalonique, elle semble trouve des appuis au sein de la Commission européenne Justice et Affaires Intérieures (JAI), qui travaille sur le sujet. À l'image de l'espace Schengen, ce dispositif pourrait être repris dans un cadre intergouvernemental afin de contourner la réticence de certains États de l'Union. À ce propos, Doede Ackers (DG JAI, Commission européenne) souligne que "c'est une analyse qui tient la route, elle doit être explorée. Des projets pilotes permettraient peut-être de confirmer les idées initiales du projet britannique ». Selon un texte de la Commission ${ }^{24}$, le système de l'asile en Europe serait menacé par « un gonflement des flux composés à la fois de personnes ayant légitimement besoin d'une protection internationale et de migrants utilisant les voies et les procédures d'asile pour accéder au territoire des Etats membres ». Johannes Van der Klaauw (HCR) va dans ce sens en précisant, lors d'un colloque européen, que «l'Europe est un peu inondée, lorsqu'on voit les chiffres ». Face à ces diverses communications discrètes et effectuées sur un mode futile, il est important de rétablir à chaque fois la réalité de la situation. Car les données statistiques contredisent cette idée généralement admise, même par le Haut Commissariat aux Réfugiés; en effet si le nombre de demandes d'asile dans les pays européens a augmenté depuis 1996, sur les quinze dernières années, on assiste plutôt à une stabilisation des effectifs, voire à une diminution durant la dernière année.

Le programme "Convention Plus» du HCR, commencé à l'automne 2002, vise à renforcer la Convention de Genève du fait des soi-disant abus de procédures de la part de «faux demandeurs » ou "réfugiés économiques ». S'adressant aux quinze pays de l'UE, le Haut Commissaire Ruud Lubbers souligne la nécessité de réfléchir à «des solutions durables dans les régions d'origine». Si cette proposition ne dénonce pas explicitement la convention de Genève de 1951, elle opte pour une stratégie de l'évitement et renforce la position restrictive des États de l'Union. «De la "protection au plus près des pays d'origine" aux camps de transit externalisés pour demandeurs d'asile, il n'y avait qu'un pas, franchi par le HCR à la fin du mois de mars 2003 » (Rodier, 2003). À la suite de la proposition britannique, le HCR renforce sa démarche en proposant des centres fermés dans les nouveaux États membres, visant à instruire les demandes des requérants ayant franchi illégalement les frontières de l'Union européenne. 
61 Ces deux projets affaiblissent l'engagement des pays de l'Union européenne pris à Tampere en 1999 d'une " application intégrale et globale de la Convention de Genève » dans le système d'asile européen. À la vue des différentes déclarations précédentes et des observations effectuées à Sangatte et dans la zone d'attente de l'aéroport de Roissy, le traitement de l'asile politique dans les pays européens risque d'être fortement mis à mal si ces différents projets aboutissent. Sans faire de généralisation, il est tout de même intéressant de rappeler que l'exercice du droit d'asile au sein de ces espaces frontaliers y est très difficile. Sur 59000 personnes ayant séjourné à Sangatte d'octobre 1999 à juillet 2002, moins de 450 demandes d'asile ont été déposées. À Roissy le taux d'admissions prononcées au seul titre de l'asile est

de 5,5\% du total des admissions en 2003 contre 20,2 \% en 2002 et $60 \%$ en 1995.

\section{Incompatibilité entre le dispositif des camps et le respect des droits de l'homme}

Ces systèmes diversifiés et parfois complexes conduisent de nombreux étrangers à vivre sur les marges des États. Ces dispositifs s'inscrivent, dans ce que Foucault définit, comme les nouvelles modalités de mobilisation et de contrôle des masses, où l'enfermement consiste à séparer de la population « saine » celles des malades placées à l'hôpital, des fous enfermés à l'asile, des délinquants emprisonnés (Di Méo, 2000 : 76). À Roissy, les étrangers qui refusent d'embarquer sont sous le coup d'une sanction judiciaire qui peut les amener à faire de quelques mois à trois années de prison ferme, assortie d'une interdiction du territoire français. Mais ces zones frontalières sont aussi devenues des sas, des interfaces, lorsque les autorités sont dans l'impossibilité de renvoyer ces étrangers. Elles laissent ainsi le champ libre à l'arbitraire administratif et élargissent celui de la précarité ; car ces « systèmes » favorisent inévitablement l'entrée dans la clandestinité de celles et ceux qui sont à la recherche d'une vie meilleure. En Angleterre, le taux d'efficacité (c'est à dire le pourcentage d'expulsés sur le nombre d'internés) varie selon les années entre 40 et $60 \%$. À Roissy, entre 1995 et 2001, il est arrivé que plus de $90 \%$ des demandeurs d'asile aux frontières entrent sur le territoire. Dans les pays d'accueil, les frontières posent donc une question fondamentale : celle de la « démocratisation des frontières » (Balibar, 1994) et du respect de l'état de droit et des conventions internationales ratifiées par ces même États. Dans l'hétérotopie que définit Foucault, chacune des frontières de l'espace (murs, intervalles, distances, alignements) a une signification anecdotique ; c'est l'espace global qu'elles produisent qui fait sens.

63 La détention n'est plus une exception en devenant un des outils de la gestion des flux migratoires. Elle contraint des étrangers à vivre un temps donné dans des zones disciplinaires, dont les souvenirs restent gravés dans la mémoire de celles et ceux qui ont transité par ces lieux. Ces situations assujettissent de plus en plus la politique d'asile à la politique migratoire. La mise en place de ces espaces frontaliers opaques est devenue "une technique brevetée, qui a donc ses inventeurs; c'est en rhétorique une argumentation; c'est aussi une fonction; c'est enfin une redécouverte " (Foucher, 1986).

\section{Conclusion}

Ainsi, depuis novembre 1938, date à laquelle le gouvernement français décida de créer des centres d'internement, et janvier 1939, date d'ouverture du premier de ces centres à Rieucros, les camps ont accueilli de nombreuses victimes des soubresauts de l'histoire 
européenne: étrangers inexpulsables, réfugiés espagnols républicains fuyant la répression franquiste en 1939, ressortissants des nations ennemies, même s'ils étaient antifascistes, au début de la Deuxième Guerre mondiale, juifs et tsiganes enfermés par la volonté du régime de Vichy, puis, après la chute de ce dernier, personnes soupçonnées de collaboration avec les Allemands, enfin, dans la période la plus récente, demandeurs d'asile en attente de réponse ou déboutés, "sans-papiers » de diverses catégories.

Le camp, présenté au départ comme un expédient provisoire, est devenu une institution quasi permanente. Les pouvoirs publics ont régulièrement peint la création de ces espaces fermés comme une nécessité : l'État, garant du bien commun et de l'ordre public, invoque son obligation d'isoler les nouveaux venus pour les surveiller efficacement et examiner leur situation administrative. Les logiques qui inspirent ces choix se révèlent diverses : républicaine entre novembre 1938 et juin 1940, vichyssoise collaborationniste de juin 1940 à l'été 1942, nazie jusqu'en 1944, épuratrice de 1944 à 1946, administrative et policière par la suite. Mais ces orientations successives procèdent d'une commune volonté politique de mise à l'écart. En effet les hôtes forcés des camps paraissent dangereux à des titres divers: les combattants des brigades internationales venus d'Espagne étaient, au lendemain de la guerre civile, parés d'une sinistre réputation qui effrayait même une partie de la gauche non communiste; les juifs possédaient, aux yeux de Vichy et des Allemands, tous les caractères raciaux et culturels qui entraînent la désagrégation de l'État; les collaborateurs internés à la Libération avaient objectivement trahi leur pays; les sans-papiers et les demandeurs d'asile sont souvent considérés comme des immigrés économiques qui se travestissent en réfugiés, des concurrents potentiels pour la main-d'œuvre nationale, des délinquants possibles en raison de leur situation clandestine.

La méfiance et la volonté d'efficacité semblent justifier dans certains cas le flou juridique, voire les violations des droits de l'homme, qui entourent la création et le fonctionnement des camps. Ceux-ci sont certes généralement organisés en vertu de textes officiels, mais ces textes émanent davantage de l'administration que du législateur. Aussi les conditions de détention apparaissent-elles très variables : en 1939, les Espagnols furent rassemblés dans des espaces encerclés de fils de fer barbelés et surmontés de miradors de surveillance; par la suite furent utilisés des bâtiments de toute nature, locaux réquisitionnés, casernes et hôtels désaffectés, baraquements édifiés à la hâte, ancienne usine comme à Sangatte. Si les Espagnols eurent le plus souvent à se plaindre des conditions alimentaires et sanitaires de leur détention, la situation empira pendant la Deuxième Guerre mondiale, période durant laquelle les camps français devinrent pour les juifs les antichambres de la mort. Au contraire, à la Libération, les camps permirent aux collaborateurs de se soustraire à la vengeance de leurs compatriotes et parfois d'échapper à une exécution sommaire. Dans le Calaisis, les hôtes de Sangatte pouvaient sortir librement, mais ils n'avaient pas la possibilité de résider ailleurs que dans le centre. Très variable aussi se révèle la durée de l'enfermement: si cette dernière est aujourd'hui réglementée par des textes, elle dépendait jadis du bon-vouloir des autorités et pouvait s'allonger indéfiniment.

La lugubre connotation du terme "camp de concentration », employé sans réserve avant 1944 et définitivement dévalorisé par la découverte des camps nazis d'extermination, a fait évoluer le concept de lieu d'enfermement et le vocabulaire. Les incertitudes juridiques ajoutent peut-être aussi à la mauvaise conscience des pouvoirs 
publics. En tout cas, ces derniers ont renoncé aux clôtures visibles et aménagent même parfois, dans la plus totale discrétion, des lieux d'internement comme celui d'Arenc découvert à Marseille en 1975. Le mot camp, désormais répudié, est remplacé par ceux de centre de rétention pour les sans-papiers et de zone d'attente pour les candidats à l'entrée.

L'adoucissement du vocabulaire s'est-il accompagné d'une amélioration des conditions de l'enferment? Certes il ne semble pas que des personnes meurent comme ce fut jadis le cas d'Espagnols blessés privés de soins ${ }^{25}$. De même les mauvais traitements endurés pendant la guerre ont disparu. Cependant des enquêtes ont montré que certains centres contemporains n'offraient pas toute garantie en matière d'hygiène et de respect des droits des personnes.

Ainsi les camps posent depuis les années trente une question à la fois politique et morale. Le principe de l'existence de tels centres n'a guère été discuté dans l'opinion et dans la classe politique. Avant la Deuxième Guerre, les communistes exigeaient bien la libération de leurs camarades espagnols, mais ils auraient volontiers enfermé leurs ennemis trotskistes, anarchistes et russes blancs. De la sorte, l'acceptation des camps ou l'indifférence à leur égard a permis aux responsables d'organiser l'institution à leur guise, en fonction des priorités politiques du moment, dans un relatif flou juridique. C'est précisément là que se situe la difficulté. En l'absence d'une forte pression de l'opinion, les pouvoirs publics ont géré les groupes et non les individus, mis plus en avant les impératifs globaux au service de l'État que la sauvegarde des valeurs humaines. Dans les sociétés organisées, les individus dangereux ou supposés tels se trouvent privés de liberté, mais il se révèle délicat de concilier l'intérêt général et le respect des droits individuels.

\section{BIBLIOGRAPHIE}

AGAMBEN Giorgio (1995) Qu'est-ce qu'un camp ? in Moyens sans fins, Paris, Payot, 153 p.

AZÉMA Jean-Pierre, BÉDARIDA François éds (1993) La France des années noires (1938-1948), Seuil, 2 vol., 1088 p.

BADIA Gilbert (1979) Les barbelés de l'exil, 1938-1940, Grenoble, Presses Universitaires de Grenoble.

BALIBAR Étienne (1994) Qu'est ce qu'une « frontière »? in Caloz-Tschopp Marie-Claire, Clevenot Axel et Tschopp Maria-Pia dir., Asile, violence, exclusion en Europe : histoire, analyse, prospective, Genève, Cahiers de la Section des Sciences de l'Éducation de l'Université de Genève Groupe de Genève «Violence et droit d'asile en Europe», pp. 335-341.

BLANCHARD Emmanuel, RODIER Claire (2003) L'Europe des camps, Plein Droit, n 58, pp. 14-17. BLOCH Marc (1949) Apologie pour l'histoire ou métier d'historien, Paris, Armand Colin. BOURDIEU Pierre (1993) Effets de lieu in La misère du monde, Paris, Le Seuil, Libre pensée. BOUTEILLET-PAQUET Daphné (2001) L'Europe et le droit d'asile, Paris, L'Harmattan, 396 p. 
BROSSAT Alain (1996) L'épreuve du désastre, le XXème siècle des camps, Paris, Bibliothèque Albin Michel Idées, $512 \mathrm{p}$.

BRUNET Roger, FERRAS Robert, THÉRY Hervé (1992) Les mots de la géographie, Paris, Reclus - La documentation française, $518 \mathrm{p}$.

CALOZ-TSCHOPP Marie-Claire (2004) Les étrangers aux frontières de l'Europe : le spectre des camps, Paris, La Dispute, 252 p.

CIMADE (1985) Les clandestins de Dieu, éd. Labor et Fides.

DEGUIGNÉ Nathalie et TÉMIME Émile (2001) Le camp du Grand Arénas, 1944-1966, Paris, Autrement, coll. Français d'ailleurs, peuple d'ici.

DI MÉO Guy (1998) Géographie sociale et territoires, Paris, Nathan Université, 320 p.

DREYFUS-ARMAND Geneviève, TÉMIME Émile (1995) Les Camps sur la plage, un exil espagnol, Autrement, coll. Français d'ailleurs, peuple d'ici, $141 \mathrm{p}$.

FILHOL Emmanuel (2004) La mémoire et l'oubli, l'internement des Tsiganes en France (1940-46), Paris, L'Harmattan, 115 p.

FISHER Nicolas (2003) L'internement républicain, Plein Droit, Des camps pour étrangers, $\mathrm{n}^{\circ}$ 58, pp. $18-21$.

FOUCHER Michel (1987), L'invention des frontières, Paris, Fondation pour les Études de Défense Nationale, coll. Les 7 épées, 325 p.

GRYNBERG Anne (1991) Les camps de la honte, les internés juifs dans les camps français (1939-44), Paris, La Découverte, 400 p.

GUILLEMOLES Alain, DOMON Arlette (1990) Aux origines de la Cimade, numéro spécial de Cimade Information, juillet-septembre.

HARRELL-BONDI Barbara et OPONDO Enoch (1996) La rétention des demandeurs d'asile dans la forteresse britannique : une évaluation, Cultures \& Conflits, $\mathrm{n}^{\circ} 23 \mathrm{http}$ ///conflits.revues.org

LABORIE Pierre (1992) L'opinion française sous Vichy, Paris, Seuil, coll. l'Univers historique, 412 p.

LAHARIE Claude (1993) Le camp de Gurs, 1939-45, un aspect méconnu de l'histoire de Vichy, Pau, $\mathrm{J} \& \mathrm{D}$ éditions $397 \mathrm{p}$.

LEGOUX Luc (2002) Vers une redéfinition du droit d'asile, Les migrations internationales, Cahiers français, Paris, La documentation française, $\mathrm{n}^{\circ} 307$, pp. 77-84.

MIGREUROP (2003) Les camps d'étrangers en Europe, http://migreurop.org

MILZA Pierre, PESCHANSKI Denis (1994) Exils et Migration: Italiens et Espagnols en France (1938-46), Actes du Colloque de Salamanque, Turin et Paris, L'Harmattan, 695 p.

NOVAK Fadela (1994) La vie dans un camp de transit tchèque, Panoramiques, pp. 81-87.

PESCHANSKI Denis (2001) Des étrangers dans la résistance, Paris, éditions de l'Atelier, Musée national de la Résistance, 126 p.

PESCHANSKI Denis (2002a) La France des camps. L'internement (1938-46), Paris, Gallimard, coll. La suite des temps, $560 \mathrm{p}$.

PESCHANSKI Denis (2002b), Autour de Sangatte, in Salmon J. Sangatte, le hangar, Bruxelles, éd. Trans Photographic, pp. 6-8. 
PESCHANSKI Denis, HUBERT Marie-Christine, PHILIPPON Emmanuel (1994) Les Tsiganes en France (1939-46), Paris, CNRS éditions, $176 \mathrm{p}$.

RAZAC Olivier (2000) Histoire politique du barbelé, Paris, La Fabrique, 111 p.

RODIER Claire (2003) Dans les camps hors d'Europe : exilons les réfugiés, Vacarme.

RUBIO Javier (1995) La politique française d'accueil : les camps d'internement, in Pierre Milza et Denis Peschanski Exils et Migration : Italiens et Espagnols en France (1938-46), Paris, L'Harmattan, 695 p.

SCHAUL Dora (1987) Un camp d'internement : Rieucros en Lozère,injoutard Philippe, Poujol Jacques et Cabanel Patrick, Cévennes, terre de refuge 1940-44, Presses du Languedoc, 379 p.

SCHOR Ralph (1996) Histoire de l'immigration en France de la fin du XIXème siècle à nos jours, Paris, Armand Colin, 347 p.

SCHOR Ralph (1985) L'opinion française et les étrangers (1919-39), Publications de la Sorbonne, $761 \mathrm{p}$. SCHRAMM Anna, VORMEIER Barbara (1979) Vivre à Gurs, Un camp de concentration français, Paris, Maspéro, coll Actes et Mémoires du peuple, $379 \mathrm{p}$.

VIRGILI Fabrice (2000) La France virile, Paris, Payot.

WEIL Patrick (1991) La France et ses étrangers, Paris, Calmann-Lévy, 403 p.

\section{ANNEXES}

\section{Carte : compléments d'information}

Ont été représentés principalement les lieux physiques d'enfermements des étrangers où ils sont totalement privés de leur liberté de circulation (" camps fermés ») à l'intérieur et aux frontières de l'espace de l'Union européenne.

Nous distinguons deux situations d'enfermement représentées :

- en blanc pour ceux qui regroupent des personnes désirant entrer sur le territoire, demandeurs d'asile ou migrants dont l'accès immédiat sur le territoire en vue d'un séjour ou d'un simple transit est conditionné à l'examen de leurs situations. À la suite de cet examen, il peut y avoir admission sur le territoire ou éloignement) ;

- en gris pour ceux qui regroupent des personnes se trouvant en situation irrégulière sur le territoire d'un État et en instance d'éloignement (pour les étrangers déboutés de l'asile et/ou du droit au séjour).

Ont été représentés en noir les lieux mélangeant ces deux fonctions (examen de l'admission/expulsion) et pouvant également servir de sas d'identification.

Des exceptions ont cependant été faites pour des situations jugées représentatives et symboliques d'une frontière qui s'externalise : c'est le cas, par exemple, pour les « camps ouverts » de Ceuta et de Melilla.

Pour certains pays, comme l'Allemagne et l'Irlande, certaines particularités ont été mises en avant : les prisons sont très souvent utilisées pour enfermer les étrangers en situation irrégulière. 
Enfin, une partie des camps cartographiés sont des lieux de regroupement informels, qui ne sont pas directement placés sous le contrôle des autorités.

- Au sud de la Méditerranée, les migrants s'y regroupent dans l'attente et pour l'organisation d'un passage en Europe.

- Dans des pays européens comme l'Italie ou la France, on retrouve ces types de regroupement dans des lieux de mise à l'écart : une gare désaffectée (Tiburtina) à Rome (où des migrants sont dans l'attente d'une régularisation), Calais (avec la présence de plusieurs centaines d'étrangers qui prévoient de passer en Angleterre).

\section{Lieux représentés :}

Le signe $\mathbb{1}$ précédant les différents noms, désigne un camp ouvert.

ALLEMAGNE : 1 Baden-Württemberg (Rottenburg, Mannheim), 2 Bayern (München, Nürnberg, Landshut, Aichach ; $\mathbb{1}$ Fürth), 3 Berlin (Köpenick), 4 Brandenburg (Eisenhüttenstadt), 5 Bremen, Oldenburg, 6 Hamburg, Lasmoor, Hahnöfersand, 7 Hessen (Offenbach, Fulda, Kassel, Frankfurt am Main, Wiesbaden, Rockenberg), 8 Niedersachsen (Braunschweig, Hameln, Hannover, Lingen, Vechta et Wolfenbüttel ; $\mathbb{0}$ Bramsche-Hesepe, Braunschweig, Oldenburg), 9 Nordrhein-Westfalen (Büren, Moers, Neuss, Köln, Wuppertal, Dortmund, Bochum), 10 Mecklenburg Vorpommen (Bützow), 11 Rheinland-Pfalz (Ingelheim, Zweibrücken, Zweibrücken/Birkenhausen, Saarbrücken et Ottweiler ; $\mathbb{1}$ Trier), 12 Sachsen (Bautzen, Chemnitz, Dresden, Görlitz, HemnitzReichenhain, Leipzig, Plauen, Stollberg, Torgau, Waldheim, Zeithain et Zwickau), 13 Sachsen-Anhalt (Volkstedt, Raßnitz et Halberstadt), 14 Schleswig-Holstein (Rendsburg, Kiel, Lübeck, Neumünster et Flensburg), 15 Thüringen ( $\mathbb{I}$ Goldlauter).

AUTRICHE : 1 Aéroport de Vienne, 2 Talham, 3 Traiskirchen, $4 \mathbb{\llbracket}$ Bad Kreuzen, $5 \mathbb{\|}$ Reichenau

BELGIQUE : 1 Merksplas (Anvers), 2 Bruges, 3 Vottem (Liège), 4 Le centre 127 (Melsbroeck) \& le 127 bis (Steenokkerzeel), 5 Aéroport de Bruxelles (Zaventem).

DANEMARK : $1 \llbracket$ Sandholm, 2 Nyborg.

ESPAGNE : 1 Algeciras (centre de rétention + Local de détention de Las Eras), 2 Barcelone (La Verneda \& Zona Franca), 3 Fuerteventura (Aéroport \& El Matorral), 4 Lanzarote, 5 Madrid (Mortalaz \& Barajas Aéropuerto), 6 Málaga, 7 Murcia, 8 Tarifa («Isla de las Palomas » de la Garde Civile), 9 Tenerife, 10 Valence, $11 \mathbb{\Downarrow}$ Ceuta Calamocarro, $12 \mathbb{\Downarrow}$ Melilla.

ESTONIE : 1 Harku.

FINLANDE : 1 Helsinki (Aéroport Vantaa), 2Katajanokka detention center.

FRANCE : Centres de rétention : 1 Bordeaux, 2 Calais-Coquelles, 3 Strasbourg Geipolsheim, 4 Hendaye, 5 Lille, 6 Lyon, 7 Marseille, 8 Nantes, 9 Nice, 10 Région parisienne (Bobigny, Le Mesnil-Amelot - Roissy, Nanterre, Paris, Versailles, Vincennes), 11 Rivesaltes, 12 Rouen, 13 Saint Louis, 14 Sète, 15 Toulouse ; Zones d'attente : 1 Bordeaux Aéroport, 2 Dunkerque, 3 Strasbourg, 6 Lyon-Saint-Exupéry, 7 Aéroport et Port de Marseille, 8 Saint-Nazaire, 9 Nice, 10 Paris (Aéroports d'Orly et Roissy), 12 Rouen, 14 Sète, 15 La Rochelle, 16 Toulon ; 17 Calais, 18 Paris (square Alban Satragne). 
GRECE : 1 Aéroport Hellénique d'Athènes, 2 Andros, 3 Chios, 4 Crète (Ierapetra, Rethymno, Sitia), 5 Région d'Evros (poste de police), 6 Githio, 7 Igoumenitsa, 8 Kalamata, 9 Karistos, 10 Kos, 11 Lerros, 12 Mykonos, 13 Mytilène - île Lesbos, 14 Naxos, 15 Rhodes, 16 Samos et Patmos, 17 Syros, 18 Zakinthos.

HONGRIE : 1 Balassagyarmat, 2 Budapest (Aéroport de Ferihegy), 3 Gyor, 4 Kiskunhalas, 5 Nagykanizsa, 6 Nyirbàtor, 7 Oroshàza, 8 Szombathely.

IRLANDE : 1 Mountjoy Prison \& Arbour Hill Prison (Dublin), 2 Cork Prison, 3 Limerick Prison, 4 Abbey Arch (Galway), 5 Aéroport de Dublin, 6 Aéroport de Shannon.

ITALIE : 1 Agrigento - San benedetto, 2 Bologne - via Mattei, 3 Brindisi Restinco, 4 Caltanissetta, 5 Milan (Via Correlli \& Aéroport), 6 Modena-Lamarmora, 7 LameziaTerme, 8 Lampedusa, 9 Lecce Regina Pacis, 10 Rome (Ponte Galleria \& Aéroport de Fiumicino), 11 Turin-Corso Brunelleschi, 12 Salina ; 13 Gare désaffectée de la Tiburtina à Rome.

LETTONIE : 1 Mucenieki, 2 Olaine.

LITUANIE : 1 Pabrade.

LUXEMBOURG : 1 Schrassig.

MALTE : Floriana, hôpital psy. Mount Carmel, Safi Barracks, Ta'Kandja ; $\mathbb{l}$ Lyster Barracks, $\mathbb{1}$ Hal Far

NORVEGE : 1 Oslo (Aéroport de Fornebu).

PAYS-BAS : 1 Aéroport Schiphol, 2 Aéroport de Rotterdam, 3 Deportation-Lodges Amsterdam, 4 Prison Noordsingel Rotterdam, 5 Ter Appel, 6 Tilburg, 7 Zeist/ Soesterberg.

POLOGNE : 1 Biala Podlaska, 2 Bielsko - Biala, 3 Chelm, 4 Elblag, 5 Gdansk, 6 Jelenia Góra, 7 Katowice, 8 Jaworzn, 9 Tychy, 10 Konin, 11 Lublin, 12 Limanowa, 13 Opole, 14 Krosno Odrzanskie, 15 Ostroleka, 16 Pila, 17 Piotrków Trybunalski, 18 Suwalki, 19 Szczecin, 20 Torun, 21 Walbrzych, 22 Wroclaw, 23 Varsovie (Chodecka-Str., Warszawa Raginis-Str., Lesznowola \& Aéroport d'Okecie).

PORTUGAL : 1 Aéroport de Lisbonne.

REPUBLIQUE TCHEQUE : 1 Balkova, 2 Cerveny Ujezd, 3 Fry'dek-mistek, 4 Postorna, 5 Praha-Ruzyné, 6 \& 7 Velké Prilepy I \& II, 8 Vysni Lhoty, 9 Aéroport de Prague-Ruzyne.

ROYAUME-UNI : 1 Campsfield (Kidlington près d'Oxford), 2 Colebrook, 3 Dover, 4 Dungavel (Strathaven, Scotland), 5 Harmondsworth (Aéroport d'Heathrow), 6 Haslar, 7 Longport, 8 Aéroport de Manchester, 9 Lindholme, 10 Oakington, 11 Queen's Building, 12 Tinsley, 13 Yarl's Wood (Clapham près de Bedford), 14 Her Majesty's Prison (HMP) Maghaberry.

SLOVAQUIE : 1 Medvedov, 2 Secovce.

SLOVENIE : 1 Ljubljana ( $\mathbb{1}$ Celovska street \& south eastern suburb), 2 Prosenjakovci, 3 Postojna.

SUÈDE : Stockholm (1 Flen, 4 Märsta, 5 Upplands Väsby), 2 Gothenburg, 3 Malmö / SUISSE *** : $1 \mathbb{d}$ Altstätten, $2 \mathbb{U}$ Bâle, $3 \mathbb{\|}$ Chiasso, $4 \mathbb{\|}$ Kreuzlingen, $5 \mathbb{U}$ Vallorbe, 6 Aéroport de Genève, 7 Aéroport de Zürich. 
ALBANIE : 1 Babrru.

BULGARIE : 1 Burgas, 2 Plovdiv, 3 Sofia, 4 Varna.

CROATIE : 1 Jezevo, $2 \mathbb{\|}$ Sasna Grada (près de Sisak).

ROUMANIE : 1 Bucarest (Aéroport d'Otopeni), 2 Arad, 3 Bucarest, 4 Galati, 5 Giurgiu, 6 Radauti.

RUSSIE : 1 Moscou (Aéroport de Chérémétiévo).

SERBIE/MONTENEGRO : Belgrade (1 Padinska Skela \& 2 motel près du mont Avala).

UKRAINE : 1 Pavshino (Mukachevo).

ALGERIE : 1 Camp de Maghnia, 2 In Guezzam, 3, Tin Zaouatine ; Alger, Tamanrasset.

LIBAN : 1 Beyrouth, 2 Roumieh ; Beyrouth (camps palestiniens).

MAROC : 1 Bel Younech, 2 El-Aioun, 3 Gourougou, 4 Messnana (la forêt de Belyounech), 5 Oujda (la forêt de Beni Issnasen), Tarfaya (6 Ras El Oued, 7 Hagounia) ; Casablanca, Fès, Rabat, Tanger.

TURQUIE : 1 Kayseri, 2 Konya, 3 Silopi, 4 Van, 5 Istanbul, 6 Yozgat ; Ankara, Istanbul, Izmir, Van.

\section{NOTES}

1. La première partie de cet article, les camps des années noires, est de Yvan Gastaut, la seconde, les camps aujourd'hui, de Olivier Clochard qui a aussi rédigé l'introduction ; la conclusion d'ensemble est de Ralph Schor.

2. Voir les travaux de Marc-Olivier Baruch sur l'Administration française en général et plus particulièrement sous Vichy.

3. Il y eut un bref intermède sous la forme d'un deuxième gouvernement Blum entre le 13 mars et le 10 avril 1938.

4. Philippe Serre était député de Meurthe et Moselle et sous-secrétaire d'État au Travail dans le troisième gouvernement Chautemps entre le 25 juin 1937 et le 18 janvier 1938.

5. Cette fonction fut éphémère car le gouvernement ne dura que du 18 janvier au 13 mars. Par la suite Philippe Serre redevint sous-secrétaire d'État au Travail.

6. Voir aussi le site sur la mémoire du camp http://gurs.free.fr

7. Voir le film de Jacqueline Veuve (1997) Journal de Rivesaltes 1941-42, Suisse, Cinémanufacture, 35 m., 77 min.

8. En Zone nord, l'administration française était malgré tout placée sous l'autorité allemande.

9. René Bousquet accepta que la police française se charge d'organiser les rafles en zone occupée tandis que Pierre Laval proposa que, dans la zone libre, les enfants de moins de 16 ans, non réclamés par les Allemands soient livrés en même temps que leurs parents.

10. Un accord avait été signé entre les Allemands Théodor Danneker représentant l'office central de sécurité du Reich, Karl Oberg, chef de la police SS en France et les Français Pierre Laval et René Bousquet.

11. Ces militants issus des mouvements de jeunesse protestants avaient fondé le «CIM » (Centre Inter-Mouvement) auquel ils rajoutèrent un « ade » : Auprès Des Évacués (Guillemoles et Domon, 1990).

12. Circulaires du 22 juin, $1^{\text {er }}$ juillet, 24 octobre et 18 novembre 1946. 
13. On dénombra jusqu'à 1500 Italiens dans ce camp situé dans un ancien hôtel désaffecté à Garavan en 1947.

14. Archive de l'UD CGT des Alpes maritimes, lettre du 14 décembre 1946.

15. Paris, CNRS, EHESS, 15-17 juin 1991.

16. «Des milliers de fantômes en camp ": Enquête sur les "réfugiés » de Sangatte réalisée en octobre 2000 par le CCFD, la CIMADE, le GISTI, le SAF et le SM.

17. Ce qui frappe dans les diverses argumentations, c'est la mise en avant de l'absence de registre. À la comparaison au camp d'internement, Denis Peschanski note qu'il existe " nul registre comme dans tous les camps d'internement: il suffit de s'y rendre pour se voir attribuer un lit. Quant à la gestion du centre, elle est dans les mains de la Croix Rouge " (Peschanski, 2002b : 6-8). Or d'après les observations effectuées, il a bien existé un enregistrement quasi officiel des personnes qui arrivaient dans le camp de Sangatte. L'historien conclut toutefois en indiquant que la référence à un système de camp n'est pas infructueuse car " elle permet de repérer (...) des comportements voisins ".

18. Tumin S. Immigration detention center - Campsfield House: report of a Unannounced short inspection by HM Inspectorate of Prisons, London, Home Office, p. 9. Cité par Harrell-Bondi et Opondo, 1995.

19. Toutefois cette euphémisation des lieux où des exilés sont retenus, semblent s'estomper sur les marges de l'Union; l'article de Fadela Novak, paru en 1994, fait référence à cinq camps d'accueil pour les réfugiés en République Tchèque; ces camps-tampons existent aussi dans les pays de la rive sud de la Méditerranée comme au Maroc ou en Algérie.

20. Dans les premiers temps, ce terme générique est employé dans l'ensemble des pays qui créent ces dispositifs aux frontières extérieures de ce qui était alors, la Communauté européenne. 21. Les camps à Malte, http://migreurop.org

22. Au-delà du $4{ }^{\text {ème }}$ jour, si l'administration souhaite renouveler le maintien en zone d'attente d'un étranger, elle doit le présenter devant cette instance judiciaire.

23. AFP, 21 juillet 2004.

24. Communication de la Commission au Conseil et au Parlement européen (2003) 152 final.

25. Depuis le milieu des années quatre-vingt dix, les violences policières, les déportations et l'absence de soin ont toutefois entraîné la mort de plus de 150 étrangers au sein de l'Union européenne. Voir carte d'olivier Clochard et Philippe Rekacewicz (mars 2004) En dix ans plus de 4 000 morts aux frontières, publiée par Le Monde diplomatique.

\section{RÉSUMÉS}

La longue histoire des camps d'internement d'étrangers en France évoque une expression spatiale du rejet de l'Autre (réfugiés, immigrés, juifs, harkis...) ; elle atteste dans le même temps d'une continuité : le lieu reste, les populations exclues passent en fonction des conjonctures politiques et économiques. Les diverses formes de gestion contemporaine (parfois brutale et peu humaine) cultivent des parentés avec le passé; bien sûr, il ne s'agit pas d'établir une simple filiation avec les premiers camps d'internement des exilés espagnols ou ceux dans lesquels le régime de Vichy enferma les juifs. Mais les dispositifs répressifs élaborés dans les années trente contre les étrangers ont influencé et dans une certaine mesure conditionné l'opinion française habituée à confondre, réfugiés, juifs et travailleurs immigrés. Après la Libération, certaines 
infrastructures ont continué d'être utilisées pour des politiques et des stratégies d'exclusion différentes.

Aujourd'hui, bien que les enjeux et les formes soient totalement différents, les phénomènes d'exclusion se sont poursuivis et ont été largement développés dans le cadre de la construction européenne. Ces diverses formes de cloisonnement spatial peuvent être considérées comme de nouveaux espaces frontaliers où l'exercice des droits de l'homme est limité et dont la terminologie est riche d'euphémisme. Cartographier l'existence de ces lieux, trace du même coup les contours d'une autre Europe : celle du rejet aussi bien à l'intérieur qu'à l'extérieur de l'Union européenne. La détention des candidats à l'exil n'est plus une exception et devient la règle pour gérer des flux massifs, une règle qu'il importe d'introduire dans la juridiction de l'Union européenne, notamment celle concernant les réfugiés.

Internment Camps for Foreigners since 1938 : Continuity and Adaptations. From the French 'Model' to the Construction of a Schengen Space

The long history of internment camps for foreigners in France evokes a spatial expression of the rejection of the 'other' (refugees, immigrants, Jews, Harkis...). At the same time, it attests to certain continuity; the space remains, the excluded populations change along with the political and economic climate. The present diverse ways of managing these spaces and migrants (sometimes brutal and little humane) cultivate links of the past. This does not, of course, mean establishing a simple relationship with the first interment camps for Spanish exiles or those used by the Vichy regime to intern Jews. But the repressive measures taken against foreigners during the 1930's have influenced and to a certain extent conditioned the French public opinion to confuse refugees, Jews and immigrant workers. Since the Liberation, certain infrastructures have continued to be used for different policies and strategies of exclusion.

Today, even though their stakes and forms are entirely different, exclusionary measures have persisted and have been widely developed in the context of European construction. These diverse forms of spatial partitioning can be considered as new border spaces where the exercise of human rights is limited and the terminology of which is rich with euphemism. To map out the existence of these places traces the contours of another Europe; one of rejection inside as well as outside the European Union. The detention of candidates for exile is no longer an exception but has become the rule in regulating massive flows, a rule that should be included into the jurisdiction of the European Union, especially that which concerns refugees.

Los campos de extranjeros desde 1938 : continuidad y adaptaciones. Del « modelo » francés a la construcción del Espacio Schengen

La larga historia de los campos franceses de internamiento de extranjeros evoca una expresión espacial del rechazo al Otro (refugiados, inmigrantes, judíos, harkis,...) y atestigua la continuidad en el tiempo de dicho rechazo: mientras el lugar "permanece», las poblaciones excluidas se suceden en función de la coyuntura política o económica. En los modelos contemporáneos de gestión de la presencia de extranjeros, en ocasiones brutales y poco humanos, se pueden encontrar lazos de parentesco con el pasado. Por supuesto, no se trata de establecer una simple filiación entre los primeros campos de internamiento de exiliados españoles y aquellos en los que el régimen de Vichy encerró a los judíos. Sin embargo, los dispositivos de represión de extranjeros elaborados durante los años treinta han influenciado, y en cierto modo condicionado, a la opinión francesa la cual confunde a menudo a refugiados, con judíos o inmigrantes. Tras la Liberación, ciertas infraestructuras han seguido siendo utilizadas en el marco de diversas políticas y estrategias de exclusión.

Hoy en día, a pesar de que los intereses y las formas son totalmente diferentes, los fenómenos de exclusión continúan existiendo y se desarrollan, en particular, en el marco de la construcción europea. Estas formas de separación espacial pueden ser consideradas como nuevos espacios fronterizos en los cuales el ejercicio de los derechos del hombre se halla limitado y en los cuales 
un gran eufemismo reina en la terminología. Cartografiar la existencia de estos lugares equivale a trazar los contornos de otra Europa: la Europa del rechazo tanto en el interior como en el exterior de la Unión Europea. El arresto de candidatos al exilio ya no constituye una excepción sino la regla que permite gestionar y controlar los flujos masivos. Es necesario que esta regla sea introducida en la jurisdicción de la Unión Europea, en particular en lo que concierne a los refugiados.

\section{INDEX}

Index géographique : France, Union Européenne

Mots-clés : camps d'internement, contrôle des frontières, réfugiés

\section{AUTEURS}

\section{OLIVIER CLOCHARD}

Doctorant, équipe MIGRINTER (Unité mixte de recherche 6588 CNRS-Université de Poitiers, MSHS, 99 av. du Recteur Pineau, 86000 Poitiers, France. Courriel : CLOCHARDOL@CCPARTHENAY.FR

\section{YVAN GASTAUT}

Maître de conférences d'histoire à l'Université de Nice-Sophia-Antipolis, Centre de la Méditerranée Moderne et Contemporaine, campus de Carlone, 98 boul. Édouard Herriot, BP 209, 06204 Nice cedex 3, France. Courriel : yvan.gastaut@unice.fr

\section{RALPH SCHOR}

Professeur d'histoire contemporaine à l'Université de Nice-Sophia-Antipolis, Centre de la Méditerranée Moderne et Contemporaine, campus de Carlone, 98 boul. Édouard Herriot, BP 209, 06204 Nice cedex 3, France. 